\title{
Broadly tunable ultrafast pump-probe system operating at multi-kHz repetition rate
}

\author{
Alexander Grupp, Arne Budweg, Marco P Fischer, Jonas Allerbeck, \\ Giancarlo Soavi ${ }^{1}$, Alfred Leitenstorfer and Daniele Brida ${ }^{2}$ (i)
}

Department of Physics and Center for Applied Photonics, University of Konstanz, D-78457 Konstanz, Germany

E-mail: daniele.brida@uni-konstanz.de

\begin{abstract}
Femtosecond systems based on ytterbium as active medium are ideal for driving ultrafast optical parametric amplifiers in a broad frequency range. The excellent stability of the source and the repetition rate tunable to up to hundreds of $\mathrm{kHz}$ allow for the implementation of an advanced two-color pump probe setup with the capability to achieve excellent signal-to-noise performances with sub-10 fs temporal resolution.
\end{abstract}

Keywords: ultrafast optics, ultrafast spectroscopy, nonlinear optics, optical parametric amplifiers

\section{Introduction}

Nonlinear optical spectroscopy is an experimental concept that is the cornerstone for the investigation of fundamental light-matter interactions and pump-probe is its workhorse implementation [1-5]. This technique allows for timeresolved experiments in which an ultrashort laser pulse excites impulsively a physical system. After a controlled delay time, a second light pulse probes the transient changes that occurred in the sample. Variations in the intensity of the light transmitted/reflected by the sample are due to induced changes in absorption or refractive index and deliver insights into the evolution of the energetic landscape within the system under study. In fact, information about relaxation dynamics and energy transfer can be acquired by a precise choice of the photon energies exploited for exciting and probing the sample [1-4]. In particular, with two-color pumpprobe experiments it is possible to study a wide range of fundamental processes like electron-electron and electronphonon interactions in solid state systems characterized by broad bands for the description of the electronic states [5-7].

${ }^{1}$ Current address: Cambridge Graphene Centre, University of Cambridge, Cambridge CB3 OFA, United Kingdom.

${ }^{2}$ This article belongs to the special issue: Emerging Leaders, which features invited work from the best early-career researchers working within the scope of the Journal of Optics. Dr Daniele Brida was selected by the Editorial Board of the Journal of Optics as an Emerging Leader.
In general, ultrafast relaxation processes can occur on a timescale that can be as short as a few femtoseconds. Therefore, ultrashort laser pulses guarantee the exceptional temporal resolution that is needed for the investigation of the fastest phenomena that follow impulsive excitation. Convenient strategies for the generation of pulses with durations of only a few optical cycles rely on nonlinear optical processes driven by mode-locked and amplified laser systems. Well established ultrafast spectroscopy setups rely on Ti:sapphire technology. These lasers, due to their broadband gain spectrum, deliver pulses with durations below $6 \mathrm{fs}$ directly from the oscillator [8,9] and of $30 \mathrm{fs}$ at the output of amplification stages. In the last few decades, optical parametric amplifiers (OPAs) driven by ultrafast Ti:sapphire lasers have been developed extensively to provide tunability beyond the fundamental wavelength [5, 10-17]. With this approach, central frequencies from the deep ultraviolet [18] to the mid (MIR) and far infrared (FIR) are possible [15, 19-23]. At the same time, the generation of broadband spectra allows compressed pulses of durations well below 10 fs [10, 24-27]. Recently, new laser amplifier systems based on ytterbium doped host materials have emerged. With an emission wavelength of $1030 \mathrm{~nm}$ those lasers can be pumped directly via laser diodes, whereas conventional Ti:sapphire amplifiers require more cumbersome pumping sources. This advantage allows manufacturers to build efficient ultrafast laser systems with small footprints, excellent stability and repetition rates in 
the multi-kHz range. Adaption of the well-known OPA schemes to the $\mathrm{Yb}^{3+}$ emission is extremely promising for the generation of ultrashort pulses in a wide spectral range [26, 28-30]. Here, we present an experimental setup that is driven by a regeneratively amplified $\mathrm{Yb}$ laser and consists of synchronized OPAs producing few-fs pulses with central wavelengths spanning from the visible (VIS) to the FIR. Stable OPA operation at high repetition rate and well-designed detection schemes enable us to perform low noise measurements for which we employ single channel detection as well as multi-channel detection based on a fast $\mathrm{Si}$ line camera resolving the full $\mathrm{kHz}$ pulse train.

\section{Experimental setup}

In order to overcome the gain bandwidth limitation of ytterbium-doped gain media, which have a typical spectral width of approximately $30 \mathrm{~nm}$ [31], the combination of an $\mathrm{Yb}$ laser with nonlinear conversion stages for the generation of ultrashort pulses is highly beneficial [26, 28, 29]. With this approach it is possible to combine the intrinsic stability of the driving source with the versatility of ultrafast OPAs. Additionally, modern Yb-based amplifier systems allow repetition rates up to hundreds of $\mathrm{kHz}$ with pulse energies large enough to pump several OPAs in parallel while Ti:sapphire setups are often limited to repetition rates around $1 \mathrm{kHz}$ or require complex cooling strategies when the average power approaches the $10 \mathrm{~W}$ level. The main reason is the simpler scalability of $\mathrm{Yb}$ systems owing to the lower quantum defect of the gain medium and the longer lifetime of the lasing transition [32]. In addition, the possibility to pump $\mathrm{Yb}$ with reliable laser diodes operating in $\mathrm{CW}$ guarantees a high level of stability of the output pulse train. In our experimental system, we employ a $10 \mathrm{~W}$ ytterbium:KGW laser from Light Conversion Ltd (PHAROS 10W) as driving source. This source has a repetition rate tunable from 1 to $600 \mathrm{kHz}$. The pulses are $230 \mathrm{fs}$ long and have an energy of $200 \mu \mathrm{J}$ below $50 \mathrm{kHz}$. This value is reduced at higher frequencies while maintaining the average output power of $10 \mathrm{~W}$. We set the typical operation regime to $50 \mathrm{kHz}$ repetition rate where the pulse energy is sufficient to comfortably pump several nonlinear frequency conversion stages in parallel. At the same time, the high counting statistics can effectively reduce the noise in comparison with $1 \mathrm{kHz}$ systems.

The output power is split equally into four branches with $50 \mu \mathrm{J}$ energy each for the operation of several synchronous OPA units as sketched in figure 1. Three noncollinear OPAs (NOPAs) provide wavelengths between $450 \mathrm{~nm}$ and $2.1 \mu \mathrm{m}$ while the fourth is used for the generation of mid-infrared radiation up to $30 \mu \mathrm{m}$ via difference frequency generation (DFG). We additionally obtain short probe pulses from a compressed white light supercontinuum (WL) generated in YAG or sapphire plates. The detailed description of the parametric stages is reported in the following section.

The output of independent OPAs can be freely combined for two-color pump-probe experiments. One beam is selected for excitation and is referred to as pump. The induced changes in the optical properties of the physical system under study are then

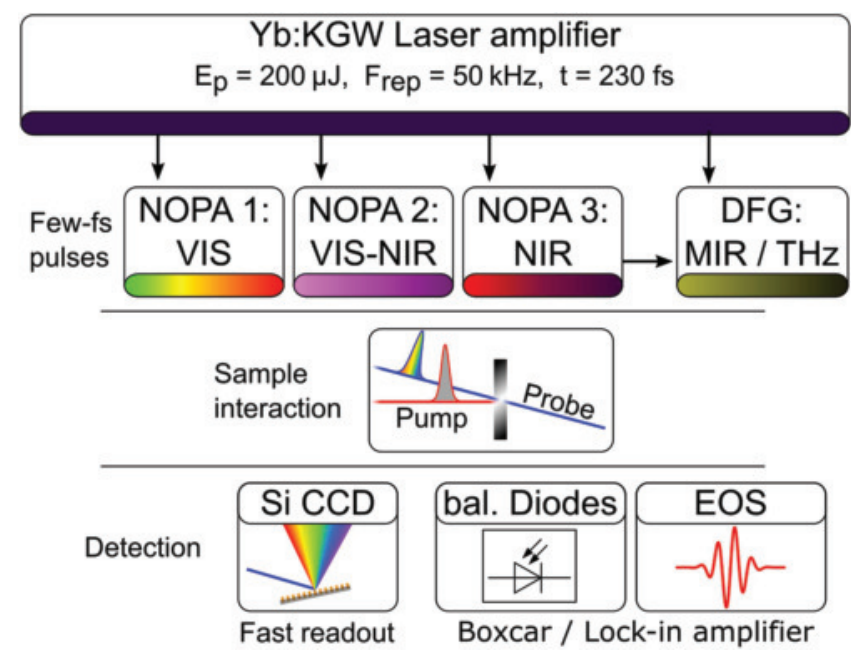

Figure 1. Schematic diagram of the experimental setup. The laser amplifier pumps three parallel OPAs and the DFG stage which can be freely combined for two-color pump-probe experiments. Detection of the probe beam is carried out at $50 \mathrm{kHz}$ with a fast siliconbased CCD spectrometer or with balanced photodiodes. Midinfrared (MIR) and far-infrared (FIR) pulses are detected via electro electro-optical sampling (EOS).

probed with a second beam. This probe beam is spatially overlapped with the first one on the sample and follows at a time delay $\tau$ that can be controlled with sub-fs precision in a correlator equipped with a motorized translation stage. The signal arises from pump induced changes in transmission or reflection of the sample. By precise selection of excitation and detection wavelengths, we match the requirements to study numerous physical systems. Importantly, at low frequencies, mechanical choppers are a convenient method to modulate the pulse train for the detection of differential signals. At higher frequencies, the mechanical modulation introduces detrimental air turbulences and is not optimal. To solve these issues, we developed a new strategy based on non-mechanical pulse modulation employing a Pockels cell (PC). This method facilitates pulse modulation with high optical contrast at frequencies up to $50 \mathrm{kHz}$.

In addition, we implemented different schemes for the detection of the probe pulses with the goal of minimizing the noise contribution to the experiments in which pump-probe signals can be limited to relative transmission changes of less than $10^{-5}$. Balanced photo diodes with a boxcar or lock-in amplifier provide a highly sensitive single channel detection scheme. With common Si or InGaAs photo diodes we cover the spectral range from $300 \mathrm{~nm}$ to $2.2 \mu \mathrm{m}$. Alternatively, pumpprobe signatures might display complex spectral features over broad frequency range. We address this experimental condition by means of a fast spectrometer. This multichannel detector is triggered by the laser system allowing us to read out one full probe spectrum every $20 \mu$ s and exploit the high correlation of subsequent laser shots to reach excellent signal-to-noise ratios. Finally, probing in the MIR/FIR spectral region can be performed by detecting the optical transients by electro-optic sampling (EOS). This time-domain scanning technique gives simultaneous access to the amplitude and phase information of the electric field of the probe pulse [33]. 
(a)

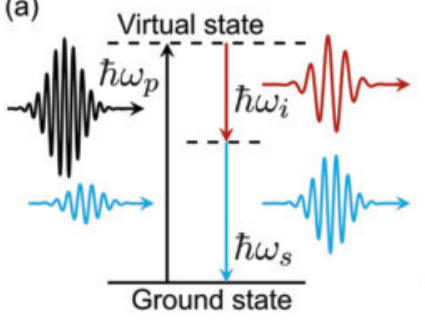

(b)

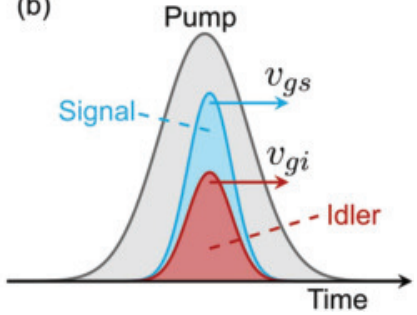

Figure 2. (a) Diagram of the energy conservation in a OPA process, involving pump $(p)$, signal $(s)$ and idler $(i)$ photons. (b) Representation of group velocity matching in a OPA.

The following sections describe in detail the nonlinear conversion stages introduced by our ultrafast setup and all the strategies developed in order to achieve the maxium sensitivity in the detection of transient signals in pump-probe experiments.

\section{Broadband sources}

Ultrabroadband OPAs are the workhorse tools used for achieving a temporal resolution of less than $10 \mathrm{fs}$ in optical pump-probe experiments. They offer enormous tunability and wide spectra which can be compressed to pulse durations as short as a few optical cycles [10, 34].

The basic concept common to typical OPAs is to provide a weak, but broadband signal beam, which is amplified in a nonlinear crystal exploiting an intense pump beam. This process is described with a second order nonlinear polarization that occurs in non-centrosymmetric crystals, which lacks inversion symmetry, and therefore exhibits a nonlinear $\chi^{(2)}$ susceptibility. Figure 2(a) sketches the energy conservation in a parametric amplification process: a high-energy pump beam $\left(\hbar \omega_{p}\right)$ excites a virtual level that is depleted by the spontaneous or stimulated emission of a signal photon $\left(\hbar \omega_{s}\right)$, energy conservation requires also the emission of a second photon defined as idler $\left(\hbar \omega_{i}\right)$. To obtain significant amplification, signal photons generated at different crystal positions need to interfere constructively. This condition requires matching of the wave vectors (phase velocities) in order to achieve momentum conservation. In common implementations, this phase matching is achieved by exploiting the birefringence of the nonlinear crystal. To achieve broadband amplification the group velocities between signal and idler have to be matched as well. This condition is met in some particular amplification conditions that exploit the refractive index dispersion of the nonlinear crystal or by introducing a non-collinearity angle between pump and signal beams [35, 36]. In this second configuration, the group velocity of the signal $\left(v_{\mathrm{gs}}\right)$ matches the projection of the idler velocity $\left(v_{\text {gi }}\right)$ in its own propagation direction as sketched in figure 2(b).

As shown in figure 3 , our home-built OPAs provide spectra ranging from $450 \mathrm{~nm}$ to $2.1 \mu \mathrm{m}$ with almost continuous coverage. Depending on the emission wavelength we employ either the fundamental frequency (FF), second harmonic $(\mathrm{SH})$, or the third harmonic $(\mathrm{TH})$ of the $\mathrm{Yb}$ laser to pump the nonlinear frequency conversion. The NOPAs in the near infrared (NIR) and visible to near-infrared spectral range (VIS-NIR) are pumped by the SH. Generation of pulses in the VIS requires pumping with the TH. Only MIR and FIR generation are carried out directly with the FF. Additionally we use up to $2 \mu \mathrm{J}$ of the FF to generate a white light supercontinuum. Those WL pulses are used for seeding of the NOPAs as well as an alternative pulse source for probing. All these parallel stages are optimized in order to deliver the shortest pulses possible at the best conditions of pulse-topulse stability and beam quality.

\subsection{Supercontinuum pulses}

Broadband spectra can be conveniently generated by exploiting the so-called white light generation that occurs when an intense pulse propagates in a dielectric medium. Third-order nonlinearity (self-phase modulation) and filamentation $[37,38]$ lead to spectral broadening. These pulses can be effectively used as a probe in ultrafast experiments or alternatively they can be amplified in OPA stages for the generation of intense pump beams.

In contrast to supercontinua obtained from photonic crystal fibers, where often significant spectral modulations occur, solid state bulk materials offer smooth and extremely broadband spectra. When driving pulse energies at the $\mu \mathrm{J}$ level are used, spectra well suited for the use in OPAs can be generated from crystals such as sapphire [39-41]. Due to the large band gap in this material it is possible to generate wavelengths approaching the UV. Additionally, damage thresholds are found to be very high which allows continuous operation without optical damage. Similar properties are also found in YAG crystals [42]. Compared to sapphire, continua from YAG crystals result in being more stable at higher repetition rates and at longer pumping wavelengths like $1030 \mathrm{~nm}$. All OPAs are seeded with a white light generated in a YAG crystal. The experimental setup for continuum generation is rather simple. A portion of the fundamental beam starting from a diameter of $2.5 \mathrm{~mm}$ full width at half maximum (FWHM) is shaped by an iris aperture and focused with a focal length of $100 \mathrm{~mm}$ into the crystal. A variable attenuator (VA) allows precise adjustment of the peak intensity at the focal spot. We obtain stable white light emission starting from $90 \mathrm{GW} \mathrm{cm}^{-2}$. Figure 4 displays two white light spectra optimized for the generation of VIS and NIR spectral components, respectively. In detail, with a peak intensity of $180 \mathrm{GW} \mathrm{cm}^{-2}$, we generate a continuum in the visible spectral range covering wavelengths from $450 \mathrm{~nm}$ to $1 \mu \mathrm{m}$ (left, blue area). Even higher peak intensities of $360 \mathrm{GW} \mathrm{cm}^{-2}$ allow pushing the short wavelength cut-off to $400 \mathrm{~nm}$ (dashed black line) at the cost of spectral intensity around $700 \mathrm{~nm}$. At intermediate peak intensities of $250 \mathrm{GW} \mathrm{cm}^{-2}$, we obtain good conditions for a supercontinuum at IR wavelengths (figure 4 right, yellow area).

Beside its purpose to seed OPAs, we also use selected spectral windows of the white light as a probe in our experiments. By separating the VIS components, we obtain a spectrum which supports a Fourier-transform-limited duration 


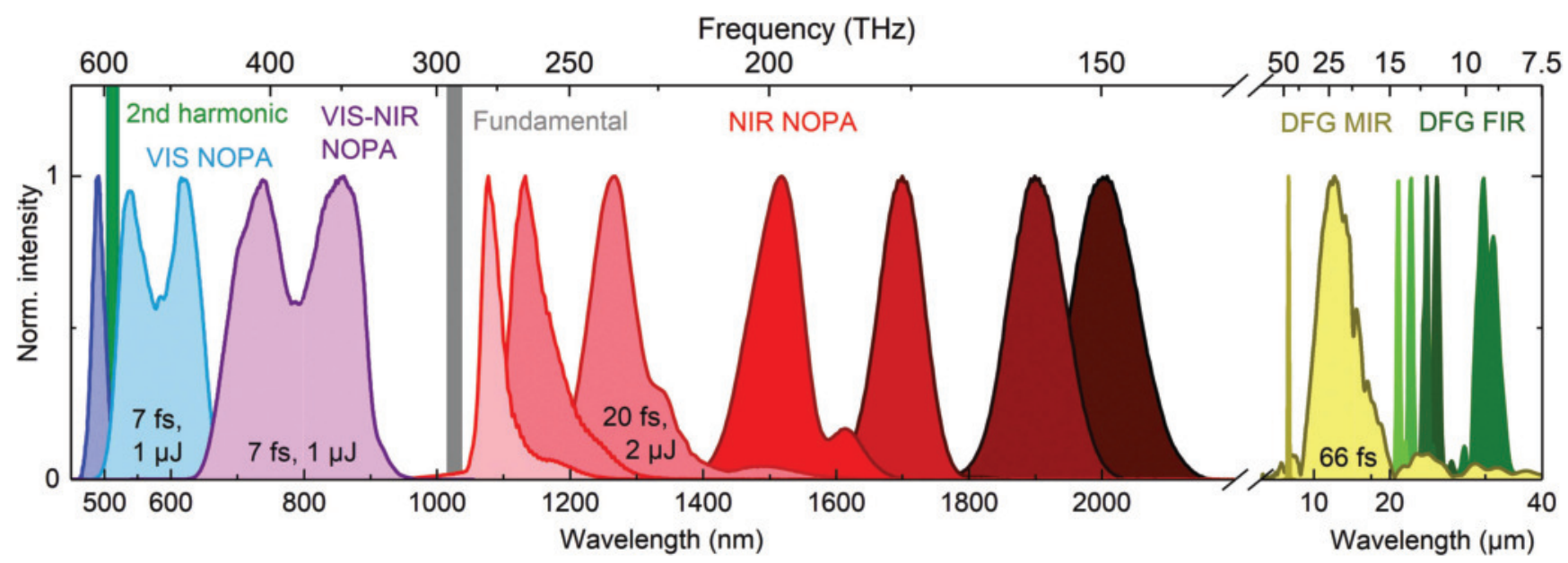

Figure 3. Intensity spectra of our home built OPAs. The combination of VIS, VIS-NIR and NIR NOPAs yields almost gapless spectral coverage from 450 to $2100 \mathrm{~nm}$. MIR and FIR pulses are obtained from DFG in GaSe and 4H-SiC of the first harmonic with the NIR NOPA. Pulse durations and pulse energies of selected configurations are displayed.

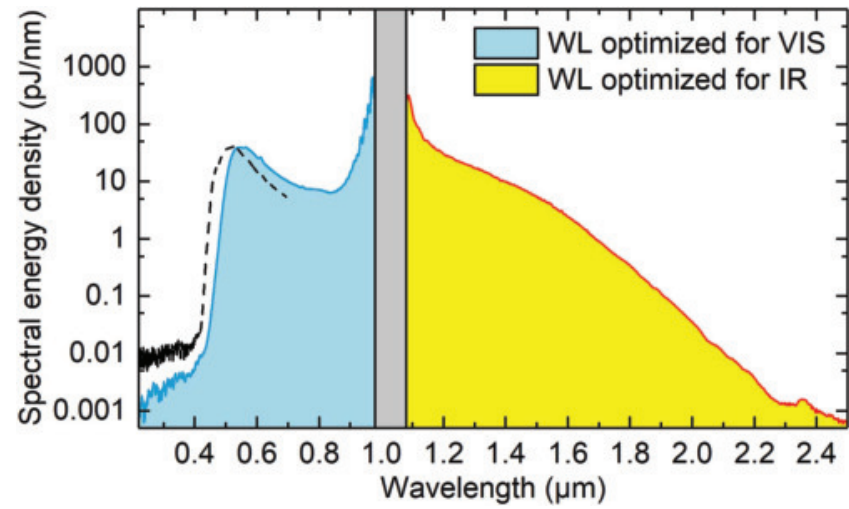

Figure 4. Spectral energy density of the white light supercontinuum generated in a $4 \mathrm{~mm}$ YAG crystal. Pump pulse energy and focusing was optimized individually for optimum conditions at wavelengths shorter (left, blue area) and longer (right, yellow area) than the fundamental wavelength. At high intensities the short wavelength cut off shifts at cost of intermediate wavelengths (dashed black). The gray area is blocked with color filters and includes the pump wavelength of $1030 \mathrm{~nm}$.

of only 3 fs. Even without any pulse compression a few-fs time resolution is possible by spectral filtering of the probe pulses prior to detection [43]. Nevertheless, compression of the probe pulses yields advantages. A pair of dielectric chirped mirrors serves for pulse compression in the spectral range from 450 to $750 \mathrm{~nm}$, where a pulse duration of approximately $7 \mathrm{fs}$ is obtained. As a result, the retrieved constant spectral phase allows the evaluation of broadband spectral signatures without any further correction of the time delay in pump-probe experiments. Also, the infrared portion of our white light is of interest for being employed as probe beam in time-resolved experiments. Here, dispersion compensation is provided by means of an SF10 prism pair with $61^{\circ}$ apex angle and a distance of $140 \mathrm{~mm}$. Short wavelength components around $1050 \mathrm{~nm}$ close to the fundamental exhibit a steeper phase as compared to longer wavelengths. For that reason, we select the spectrum with a razor blade placed in the Fourier plane of the prism compressor. Pulse characterization

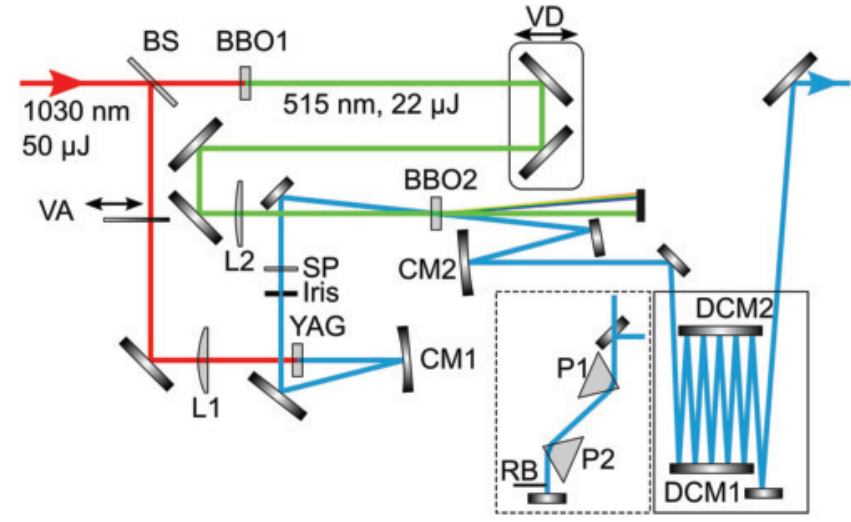

Figure 5. Schematic of the VIS-NIR NOPA. After non-collinear superposition of pump (green) and signal beam (blue) we use a pair of dielectric chirped mirrors (DCM) or alternatively a prism compressor (dashed box) for pulse compression.

is carried out via second-harmonic-generation frequencyresolved optical gating (FROG) [44] and yields pulse durations of 14 fs whereas the full IR spectrum shown in figure 4 possesses a transform limited duration of $9.5 \mathrm{fs}$. Beside pumpprobe experiments, these pulses are well suited to be employed as gates in electro optical sampling. We use them for field resolved detection of $\mathrm{THz}$ pulses with frequency components up to $50 \mathrm{THz}$.

\subsection{Generation of pulses in the visible to NIR}

The basic layout of the NOPA follows the traditional schemes as used with Ti:sapphire systems [10, 24, 26, 34]. Figure 5 presents a schematic representation of the VIS-NIR NOPA. We split a small portion from the $50 \mu \mathrm{J}$ fundamental beam for supercontinuum generation in a $2 \mathrm{~mm}$ thick YAG crystal. With a VA we tune the pulse energy to achieve optimum conditions. The white light is collimated by a curved mirror (CM1) and spectrally filtered with a short pass filter (SP) at $900 \mathrm{~nm}$ to avoid amplification of spurious wavelengths. It should be remarked that the filamentation process produces 


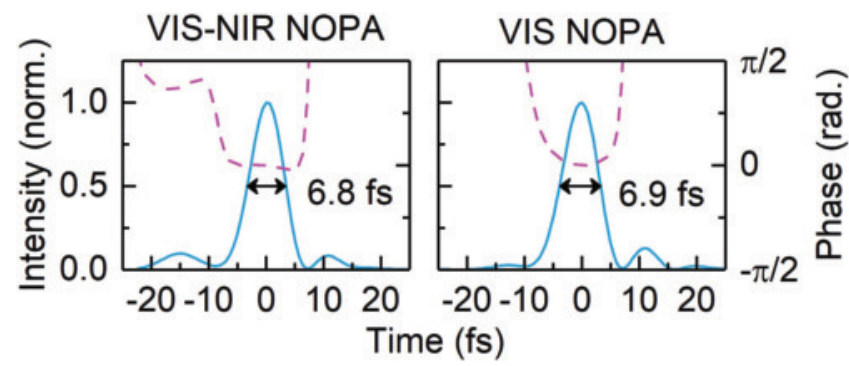

Figure 6. Reconstructed temporal profile (blue, solid) and phase (pink, dashed) from SHG- FROG traces of the VIS-NIR NOPA and VIS NOPA.

spectra that are not well-behaved in the proximity of the driving wavelength.

The main portion of the pump light is frequency doubled in a BBO crystal. For this task, we exploit type-I phasematching $\left(\theta=24^{\circ}\right)$ in a $2 \mathrm{~mm}$ thick crystal. After rejecting the residual fundamental wavelength with dielectric mirrors, we obtain $22.5 \mu \mathrm{J}$ pulse energy at $515 \mathrm{~nm}$ with a $50 \%$ conversion efficiency for pumping the OPA. A variable delay line adjusts the timing between the pulses. The pump beam is then focused with a focal length of $200 \mathrm{~mm}$. We set the focal plane several centimetres before the nonlinear medium yielding a peak intensity of $130 \mathrm{GW} \mathrm{cm}^{-2}$ inside the $1.5 \mathrm{~mm}$ thick BBO crystal, which is used for the mixing process. Broadband amplification is achieved at the phase matching angle of $\theta=24^{\circ}$ when pump and signal beams form an internal angle of $\alpha=2.6^{\circ}$. The amplified signal is collimated by a second curved mirror (CM2). Finally, we use dielectric chirped mirrors (DCM1 and DCM2) for dispersion compensation. The resulting spectrum is shown in figure 3 as VIS-NIR NOPA. We characterize the temporal profile of our pulses by means of SHG FROG and obtain a duration of below 7 fs as depicted in the reconstructed intensity profile (figure 6). The transform-limited duration is 5.6 fs.

\subsection{Generation of pulses in the NIR}

With slight modifications of the experimental layout presented in figure 5 we can adapt the NOPA spectrum to the NIR spectral region. Optimum conditions for white light generation at $1.2 \mu \mathrm{m}$ are found when using a $5 \mathrm{~mm}$ thick YAG crystal. Spectral selection with a long pass filter at $1064 \mathrm{~nm}$ allows us to exploit a broad frequency range generated by the WL process.

The pump branch is frequency doubled as described above but we reduce the peak intensity inside the parametric conversion $\mathrm{BBO}$ to $50 \mathrm{GW} \mathrm{cm} \mathrm{cm}^{-2}$ by extending the focal length to $300 \mathrm{~mm}$. The NOPA still operates with $10 \%$ signalto-pump efficiency in this condition and exhibits improved long-term stability. Broadband amplification occurs at the phase matching angle of $\theta=23.1^{\circ}$ when pump and signal beams form an internal angle of $\alpha=2.6^{\circ}$. By slight tuning of phase matching and non-collinearity angles we access spectral components from $1070 \mathrm{~nm}$ to $2.1 \mu \mathrm{m}$ as shown in figure 3 as NIR NOPA. For pulse compression, we use SF56 glass Brewster prisms, cut at an angle of $59.4^{\circ}$ with an apex (a)
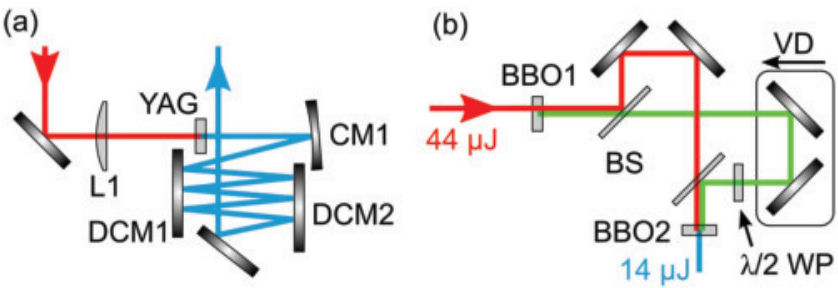

Figure 7. (a) Temporal compression of white light pulses with dielectric chirped mirrors (DCM) prior to the parametric amplification stage allows for the generation of extremely broadband VIS pulses. (b) Third harmonic generation with $32 \%$ efficiency via two separate conversion steps. Frequency doubling (BBO1) is followed by sum-frequency generation (BBO2).

distance of $220 \mathrm{~mm}$. A SHG FROG characterization yields $20 \mathrm{fs}$ pulse duration at $1300 \mathrm{~nm}$ central wavelength.

\subsection{Generation of pulses in the visible}

Accessing spectral components in the VIS range adds slightly more complexity to the setup of the NOPA [26]. For the seed generation, we focus the fundamental wavelength with a focal length of $100 \mathrm{~mm}$ into a $3 \mathrm{~mm}$ thick YAG crystal. The resulting supercontinuum extends to wavelengths as short as $450 \mathrm{~nm}$ but is strongly chirped. Prior to amplification, we therefore precompress the white light with specially designed dielectric chirped mirrors (figure 7(a)).

This NOPA is pumped by the TH of the fundamental wavelength. Figure 7(b) depicts the pump conversion setup. In a first BBO crystal, we generate the SH similar to what discussed previously. We then separate the FF and SH components by means of a dichroic beam splitter, rotate the polarization of the $\mathrm{SH}$ by $90^{\circ}$ and overlap both beams in a second BBO crystal of $2 \mathrm{~mm}$ thickness cut for type-I phase matching $\left(\theta=32.5^{\circ}\right)$. In this branch, we achieve $14 \mu \mathrm{J}$ pulse energy at $343 \mathrm{~nm}$ wavelength with an upconversion efficiency of $32 \%$. For the parametric amplification stage, the pump beam is focused with an $f=200 \mathrm{~mm}$ lens and the BBO crystal is placed $30 \mathrm{~mm}$ behind the focal plane. The pumpsignal angle is adjusted to $4.5^{\circ}$ where we obtain broadband amplification with type-I phase matching $\left(\theta=37^{\circ}\right)$. The resulting spectrum is depicted in figure 3 as VIS NOPA. By means of a second pair of chirped mirrors, we compress the NOPA pulses. Figure 6 depicts the reconstructed intensity profile where a pulse duration below $7 \mathrm{fs}$ is obtained while a Fourier-limited duration of $5 \mathrm{fs}$ is supported by the spectrum.

\subsection{Generation of pulses in the MIR}

One intuitive way to generate MIR wavelengths is to exploit the idler wave of an OPA $[12,45]$. A second route employs DFG from two NIR pulses [20,46]. Both approaches can rely on crystals like gallium-(II)-selenide (GaSe) since it offers a large transmission bandwidth in the MIR, a high second-order nonlinear coefficient and the possibility for birefringent phase matching which render it perfect for MIR frequency mixing [47]. 


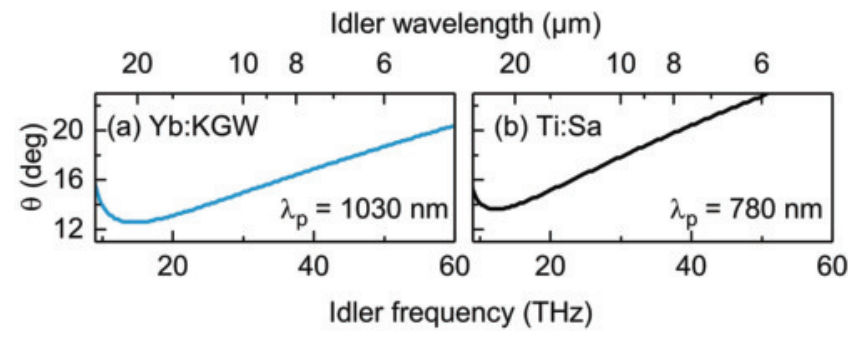

(c) $\quad 1030 \mathrm{~nm}$

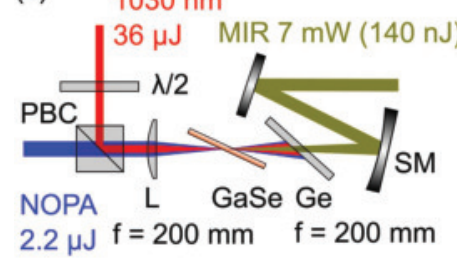

(d)

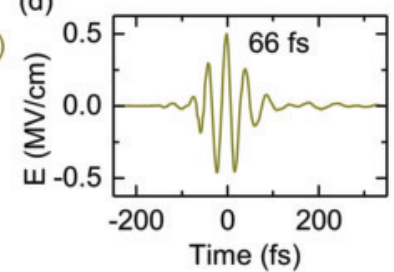

Figure 8. (a) Phase matching angle $\theta$ for DFG of the FF with the NIR NOPA in a gallium-selenide crystal (GaSe). (b) Tuning range when the fundamental frequency of Ti:sapphire is used. (c) Scheme for generation of MIR pulses via DFG. Residual NIR components are filtered with a germanium wafer (Ge). (d) Electric field of the MIR pulses reconstructed by electro-optical sampling. Peak fields of $0.5 \mathrm{MV} \mathrm{cm}^{-1}$ are obtained when focusing the pulses with an all reflective objective to a spot size of $20 \mu \mathrm{m}$.

To obtain a high conversion efficiency, we implement the approach of DFG inspired by multi-THz generation driven with Ti:sapphire systems [48]. In detail, we can drive the DFG process between the fundamental wavelength of our laser system at $1030 \mathrm{~nm}$ and the spectrum generated from the tunable NIR NOPA. With this scheme we can generate MIR wavelengths spanning from 7.5 to $30 \mu \mathrm{m}$ (10 to $20 \mathrm{THz}$ ). Calculations for type-II phase matching (figure 8(a)) display the tuning range of the DFG driven at $1030 \mathrm{~nm}$ compared to pumping with the Ti:sapphire wavelength at $780 \mathrm{~nm}$ (b). Remarkably, the red-shifted FF of the Yb laser allows for a broader phase matching condition as highlighted by the shallow dispersion of the phase matching angle in particular between 10 and $20 \mathrm{THz}$. In light of this favorable condition, a scheme requiring DFG between two NIR OPAs is not a significant improvement and would be intrinsically less efficient.

Within our experimental implementation, we overlap the FF $(36 \mu \mathrm{J}$ at $1030 \mathrm{~nm})$ and the output of the NIR NOPA $(2.2 \mu \mathrm{J})$ tuned at approximately $1200 \mathrm{~nm}$ with a polarizing beam splitter to be perfectly collinear. We focus the beams with an $f=200 \mathrm{~mm}$ lens, whereas the nonlinear crystal is placed $55 \mathrm{~mm}$ behind the focal plane. For the generated pulses, GaSe crystals of thicknesses between $100 \mu \mathrm{m}$ and $1 \mathrm{~mm}$ allow to trade-off large bandwidth with shorter crystals against intensity in longer ones. Figure 3 reports the spectra (solid green) that can be obtained by tuning the OPAs central wavelength and thus the DFG frequency. With EOS, we reconstruct the electric field of the multi-THz pulses. As a gating pulse, we employ the infrared components of our white light supercontinuum. After pulse compression, durations of $14 \mathrm{fs}$ are achieved, which allows to sample frequencies up to $50 \mathrm{THz}$. Figure $8(\mathrm{~d})$ depicts a typical Fourier-limited pulse with a duration of $66 \mathrm{fs}$ FWHM and peak electric fields of $0.5 \mathrm{MV} \mathrm{cm}^{-1}$ in the focal plane when focusing to a spot size of $20 \mu \mathrm{m}$. Spectral regions below $15 \mathrm{THz}$, where phonon resonances in most crystals hinder frequency mixing, can be reached by using silicon carbide $(4 \mathrm{H}-\mathrm{SiC})$ [49] as nonlinear emitter.

\section{Pump-probe system}

The transient absorption/reflection experiments are carried out in a two color interferometer. We evaluate differential changes in the intensity of the probe pulses transmitted (or reflected) by the pumped and un-pumped sample: $\frac{I_{\text {pumped }}-I_{\text {unpumped }}}{I_{\text {unpumped }}}=\frac{\Delta I}{I}$. Such a signal is then measured as a function of the delay between the two pulses. The differential changes usually amount to less than one percent of the probe pulse intensity. Thus, a detection scheme based on a modulation technique is needed in order to resolve the small signals. A common realization periodically blocks the pump pulses and by this imprints the signal onto the probe pulse train at the frequency of the pump modulation.

Pulse-to-pulse correlations are high when comparing subsequent transients of a pulse train and decrease when pulses are separated by multiple periods of the repetition rate. We therefore introduce a scheme for fast modulation at half of the repetition rate based on a PC. With this modulation, we evaluate subsequent laser shots of the probe $\left(I_{j+1}-I_{j}\right) / I_{j}$ and average the differential signal.

The concept for this modulation is to rotate the polarization plane of every second pulse and separate them with a polarization-dependent beam splitter. However, due to the dispersion of the nonlinear crystals an efficient half-wave operation is not possible with broadband spectra as the ones generated in the NOPAs. Placing the PC in the fundamental beam before the NOPA leads to nonlinear effects, such as self-phase modulation and self-focusing in the cm-long electro-optic crystal due to the high pulse energies and can ultimately lead to optical damage. We circumvent this problem by positioning the PC directly in front of the white light generation as shown in figure 9(a). There the pulse energies are moderate and the narrowband spectrum allows proper half-wave operation. The cell contains two potassium titanyl phosphate (KTP) crystals with $10 \mathrm{~mm}$ thickness each and is operated with a half-wave voltage of $2.4 \mathrm{kV}$. We generate the high-voltage signal from a trigger that is directly locked to the laser repetition rate. Any phase slips or beating is intrinsically avoided. Influences of the KTP dispersion on the fundamental beam are negligible since pulse broadening in the crystal amounts to less than $1 \%$ of the initial temporal duration of 230 fs. The red graph in figure 9(c) shows the modulated pulse train detected by a photo diode. We obtain an intensity contrast of 1:50. Due to the highly nonlinear behavior of the supercontinuum generation, this ratio is enough to switch off the white light generation process completely. A measurement of the pulse intensity at the sample position, depicted in the right panel of figure 9(c), shows evidence that also the 

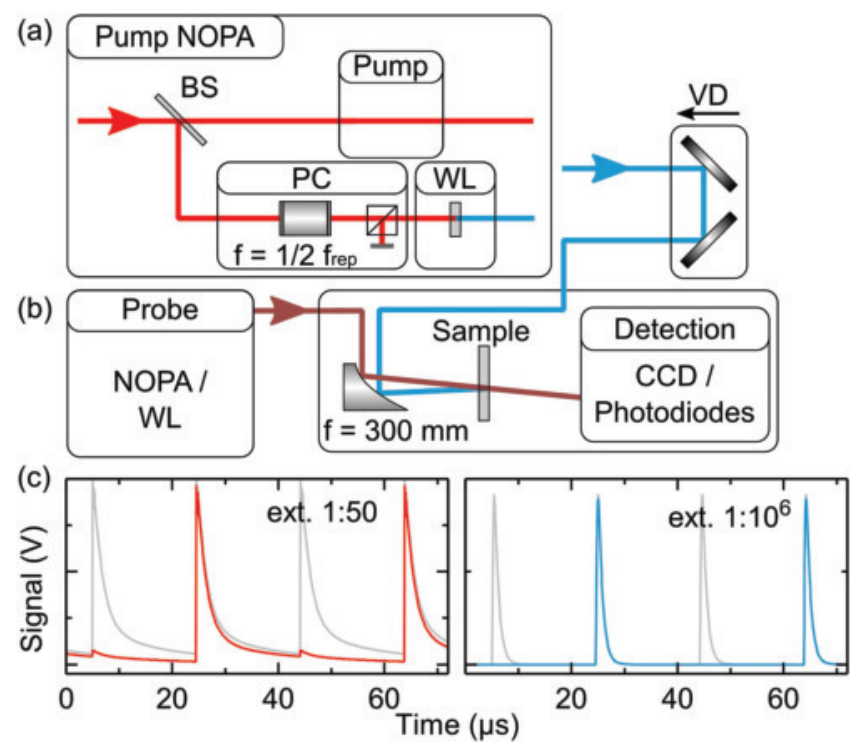

Figure 9. Schematic showing a NOPA with a Pockels cell (PC) for fast modulation at $f_{\text {rep }} / 2$ of the seed beam operating as pump branch (a) and a NOPA/white light probe (b) used for transient absorption experiments. (c) Oscilloscope measurements of the modulated pulse train before the white light generation (left, red) and at the sample position (blue, right) with an extinction factor exceeding $10^{6}$.

parametric superfluorescence in the unseeded NOPA is negligible. We obtain a contrast better than $1: 10^{6}$ limited only by the resolution of the oscilloscope used for this characterization. Compared to conventional modulation techniques such as mechanical choppers, we achieve modulation frequencies up to the repetition rate of our laser $(50 \mathrm{kHz})$ and avoid any moving components in the beam path that could lead to additional noise.

When planning an experiment, we place the $\mathrm{PC}$ in the NOPA used for excitation. Probe pulses can be supplied from a second NOPA or the compressed white light source. Both pulses are coupled into the two branches of the correlator. With curved mirrors, we reduce the spot size to approximately $100 \mu \mathrm{m}$ and overlap both beams at the sample position. By using all reflective optics, we maintain the ultrashort pulse durations. A small angle between pump and probe allows convenient spatial filtering of the pump pulses after the sample without affecting the temporal resolution.

\subsection{Noise performance and single-channel detection}

In order to be able to detect small signals, we carefully minimized the noise contributions in the generation of the NOPA pulses and in the detection used in pump-probe experiments. For white light generation precise adjustment of the laser intensity and mode shape are crucial to obtain stable emission. In optimal conditions, the WL displays rms fluctuations of $0.12 \%$, measured over 20 min with a detection bandwidth of $50 \mathrm{kHz}$, i.e. recording every single laser shot with a boxcar integrator. Figure 10(a) shows the relative energy of each laser pulse in the white light measured after spectral filtering at $550 \mathrm{~nm}$ with an optical bandwidth of $14 \mathrm{~nm}$. This characterization demonstrates that the WL is also
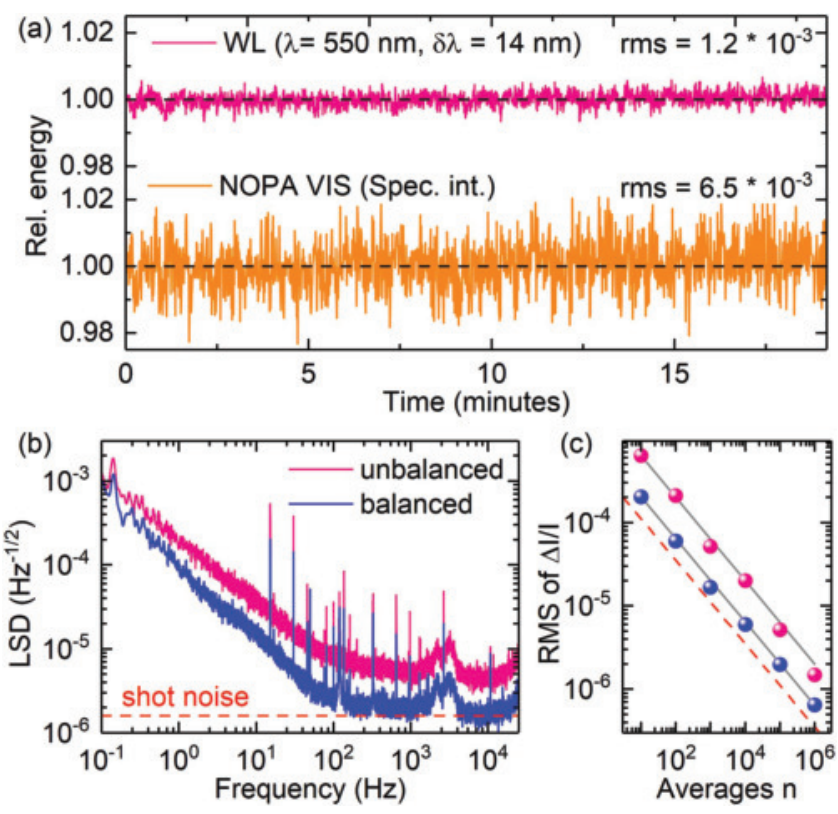

Figure 10. (a) Relative pulse energy as a function of time for the white light (WL) and the VIS NOPA. (b) Linear spectral density (LSD) normalized to the average power for the white light probe in single beam (unbalanced) and balanced (balanced) configuration. (c) Rms of the differential signal $\Delta I / I$ as a function of averages for both probe configurations. Solid lines represent $1 / \sqrt{n}$ fits indicating good statistical averaging possibilities. The shot noise level is marked as dashed line.

spectrally stable. Results obtained with the multi-channel detection confirm the wavelength independent noise level. The lower graph in figure 10(a) depicts the spectrally integrated measurement of the VIS NOPA where we observe a slightly higher average noise value of $0.65 \%$ rms fluctuations. Both graphs show an excellent long term stability, which enables us to perform data acquisition and averaging over several hours.

To improve signal-to-noise even further, we employ a balanced detection scheme that suppresses the laser fluctuations down to the shot noise limit. Therefore, a portion of the probe beam is split off prior to interaction with the sample. We pass this reference beam at a temporal offset through the sample and through the same monochromator as the probe beam. Afterwards we detect both probe replicas with a balanced circuit in which two diodes are wired for subtracting their photocurrents. Technical noise contributions originating from the laser system cancel whereas the pump-probe signal remains. With the reference beam included, the noise floor of our white light probe drops to $3.7 \times 10^{-4} \mathrm{rms}$ in the relative amplitude of the pulses. The average energy of $45 \mathrm{pJ}$ yields a shot noise level of $3.6 \times 10^{-4} \mathrm{rms}$. Figure 10(b) depicts the linear spectral density (LSD) normalized to the average power of the probe beam. We observe an improvement due to the balanced detection over the entire spectral range. At frequencies above $500 \mathrm{~Hz}$, our detection becomes limited by the shot noise of the photo current. A narrow band detection like a lock-in amplifier is therefore best used with high modulation frequencies which can be realized with the PC modulation scheme. Figure $10(\mathrm{c})$ displays the differential signal $(\Delta I / I)$ of 
(a)

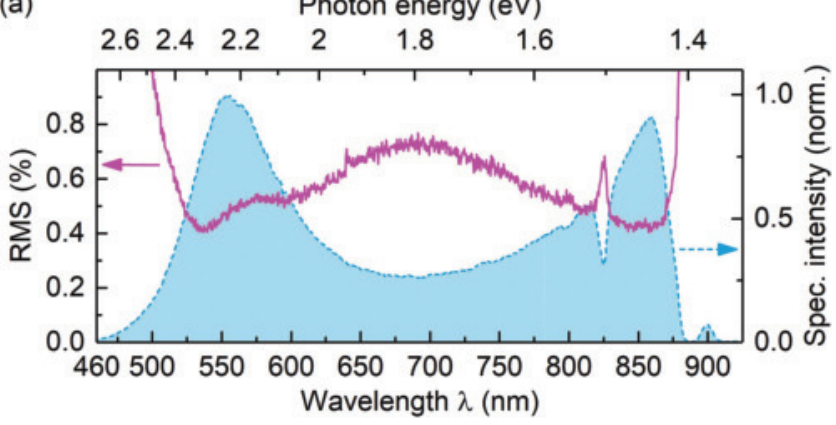

(b)

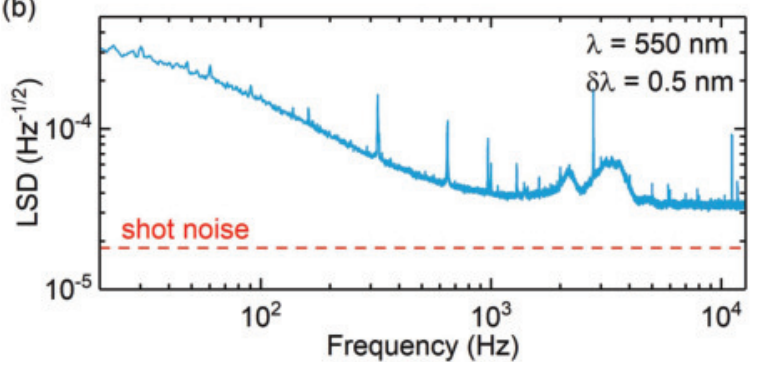

Figure 11. (a) Intensity spectrum of the white light probe recorded with the fast spectrometer (dashed blue) and rms fluctuations at each pixel (pink). (b) Linear spectral density normalized to the average power for the white light probe detected with the fast spectrometer.

an experimental pump-probe run with $10^{6}$ averages. With and without the balanced detection, the differential signal decreases with the root of evaluated averages indicated by the $1 / \sqrt{n}$ fits displayed as gray lines. We are able to reach a noise level of $6.5 \times 10^{-7}$ with our white light probe.

\subsection{Multi-channel detection}

For studies which involve rich spectral signatures, it becomes time consuming to sample broadband probe spectra with a monochromator. Enormous improvement is achieved with a multi-channel detection. For this purpose, we employ a specially developed spectrometer (Entwicklungsbüro G. Stresing, Germany) which consists of a polychromator (Acton SP2156) in combination with a fast silicon CCD detector (Hamamatsu S11490). The detector is triggered by the laser system and it is possible to read out spectra with a rate of up to $50 \mathrm{kHz}$ at a maximum modulation rate of $25 \mathrm{kHz}$. This fast acquisition works hand in hand with the pump beam modulation achieved by the PC. It allows us to subtract consecutive spectra to retrieve a wavelength dependent pumpprobe signal. Figure 11(a) shows the normalized intensity spectrum of the WL probe as detected by the camera. Its pulse energy ensures sufficient illumination of our sensor so that the supercontinuum can be efficiently used for probing. This approach is slightly advantageous compared to a NOPA pulse probe as the latter exhibits slightly higher fluctuations and lower long term stability. With optimum illumination of our sensor, we observe rms fluctuations of $0.4 \%$ on a single pixel. One pixel corresponds to an optical bandwidth of $0.5 \mathrm{~nm}$ and this detection schemes approach exceeds the shot noise limit by less than a factor of two. The noise level at different detection wavelengths corresponds to the intensity at the

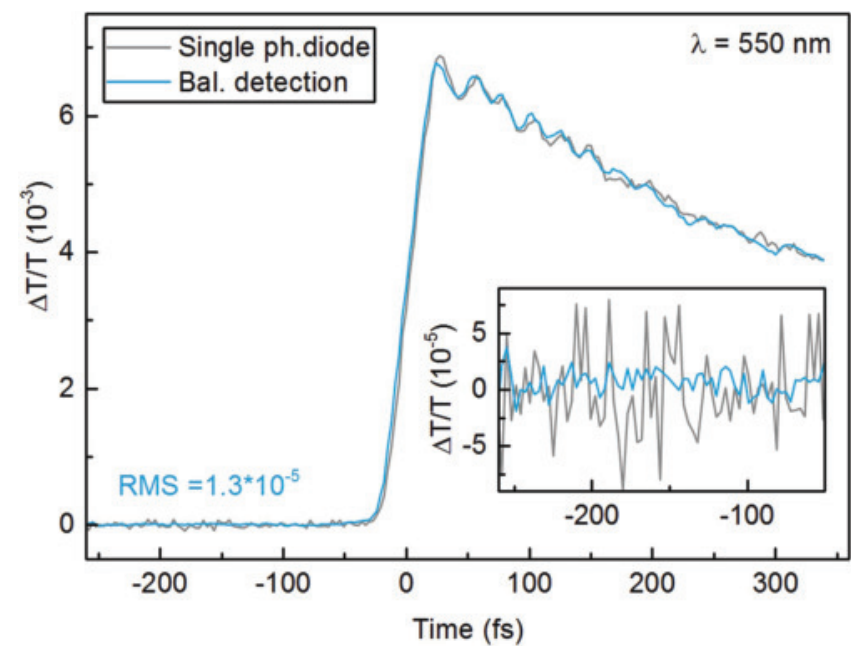

Figure 12. $\Delta T / T$ signal as function of time delay after averaging 1000 pump-on pump-off pairs of the white light probe. The noise level decreases to $1.3 \times 10^{-5}$ by including the reference beam for the balanced detection. The whole scan is performed in approximately $10 \mathrm{~s}$ in both configurations.

individual pixels and is therefore dominated by the technical noise of the CCD. Spectral fluctuations in the supercontinuum are negligible. In situations where spectral resolution is not required to be in the sub-nm range, averaging over adjacent pixels reduces the noise level. The LSD plot in figure 11(b) is again normalized to the average power and is acquired at the pixel corresponding to $550 \mathrm{~nm}$ of our probe spectrum. The noise floor is minimal at modulation frequencies higher than several $\mathrm{kHz}$. In this situation, it is again favorable to perform the evaluation of the differential pump-probe signal at the high modulation rate provided by the $\mathrm{PC}$.

\subsection{Ultrafast spectroscopy}

In this subsection, we demonstrate the use of the experimental apparatus described above for tracking ultrafast phenomena in condensed matter systems. The model sample is a $30 \mathrm{~nm}$ thick layer of poly(3-hexylthiophene) (P3HT) spin-coated on a thin fused silica substrate. This conjugated polymer is often used for opto-electronic applications like organic photovoltaics [50] and has been extensively studied in ultrafast experiments. In detail, P3HT possesses a broadband optical absorption in the visible spectral range which can be excited impulsively by pulses from the short VIS NOPA. The following dynamical evolution of the system is characterized by ultrafast relaxation and coherent phonons which allow us to assess the capabilities of the experimental setup.

First, we discuss results obtained with the single channel detection using the monochromator, balanced photodiodes and detection via the boxcar gated integrator. Figure 12 shows the differential transmission change when the polymer is excited with the VIS NOPA centered at $577 \mathrm{~nm}$. We probe with white light pulses generated in a sapphire plate and set the monochromator to $550 \mathrm{~nm}$. The graph displays the differential transmission signal as a function of the pump-probe delay after averaging 1000 consecutive pump-on and pump-off pairs for 

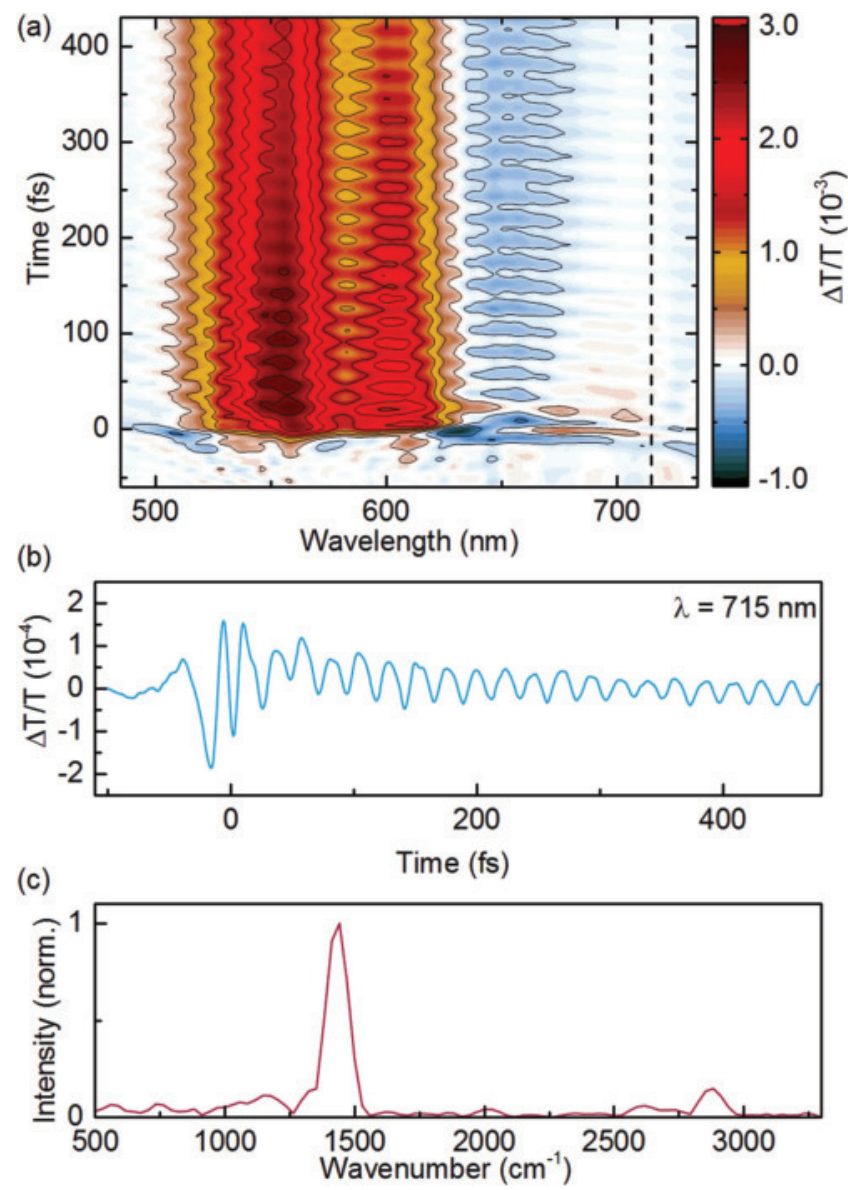

Figure 13. (a) $\Delta T / T$ signal as a function of time delay and probe wavelength. (b) Dynamics at $\lambda=715 \mathrm{~nm}$ display a fast oscillatory behavior. (c) Fourier transform of (b) as function of wavenumber showing two pronounced peaks at 1450 and $2900 \mathrm{~cm}^{-1}$ which can be assigned to vibrational modes of the excited polymer.

each temporal data point. A zoom into the signal at negative delay times clearly shows the difference between the single diode and the balanced detection. With balancing, we observe rms fluctuations of $1.3 \times 10^{-5}$ in contrast to $3.7 \times 10^{-5}$ with a single diode. At positive delay times, our signal is modulated and exhibits an oscillatory component with a periodicity of 23 fs. We attribute this signal to a coherent $\mathrm{C}=\mathrm{C}$ stretching vibrational mode of the polymer [51], which can only be excited and detected due to the high overall temporal resolution guaranteed by the ultrashort pulses employed in the experiment.

Furthermore, we use the same P3HT film to demonstrate the capabilities of the probe detection with the fast spectrometer. The sample is excited with the VIS NOPA centered at $577 \mathrm{~nm}$ and transmission changes are probed again with a white light supercontinuum. Figure 13(a) shows the retrieved differential transmission as a function of time delay and probe wavelength. Spectra were recorded at 200 different time delays between pump and probe pulse and the total data acquisition took about one hour. At wavelengths longer than $700 \mathrm{~nm}$ we observe small pump-probe signatures of $1 \times 10^{-4}$ whereas the noise floor is kept below $10^{-5}$ (see figure 13(b)). Even with such low signals, we extract two distinct peaks from the Fourier transform (figure 13(c)) of the oscillatory pattern that unveil coherent phonons up to frequencies of approximately $2900 \mathrm{~cm}^{-1}$ (symmetrical $\mathrm{CH}_{2}$ stretching mode [51]) assessing a temporal resolution of $10 \mathrm{fs}$.

\section{Conclusion}

We presented an advanced spectroscopy system that fully exploits the advantages of $\mathrm{Yb}$ laser systems for achieving $10 \mathrm{fs}$ temporal resolution in pump-probe experiments requiring exceptional low noise levels. In particular, we demonstrate that it is possible to design a class of broadband OPAs which generate pulses covering a wavelength range from 450 to $2100 \mathrm{~nm}$ and also span the mid and FIR. These sources are intrinsically synchronized allowing for multi-color spectroscopy at high repetition rates. The clear benefits of this system are its versatility and its capability to detect small signals as the ones arising from isolated quantum objects or layered materials. High frequency modulation, noise suppression, broadband nonlinear conversion stages, and sub-10 fs temporal resolution are the fundamental ingredients that will push ultrafast spectroscopy to investigate even more subtle phenomena at the core of light-matter interaction.

\section{Acknowledgments}

Support by the Emmy Noether Programm of the DFG (BR 5030/1-1), by the European Union's Seventh Framework Programme (grant agreement No. 613055 'Gemini') and by the European Research Council (Advanced Grant No. 290876 'UltraPhase') is gratefully acknowledged. We thank Stefan Eggert for developing the electronic circuits for pulse modulation and detection. We also thank Philipp Ehrenreich for the preparation of the P3HT polymer thin film.

\section{ORCID iDs}

Daniele Brida (1D https://orcid.org/0000-0003-2060-5480

\section{References}

[1] Knox W H, Hirlimann C, Miller D A B, Shah J, Chemla D S and Shank C V 1986 Femtosecond excitation of nonthermal carrier populations in GaAs quantum wells Phys. Rev. Lett. 56 1191-3

[2] Laubereau A, von der Linde D and Kaiser W 1972 Direct measurement of the vibrational lifetimes of molecules in liquids Phys. Rev. Lett. 28 1162-5

[3] Shank C V, Fork R L, Leheny R F and Shah J 1979 Dynamics of photoexcited GaAs band-edge absorption with subpicosecond resolution Phys. Rev. Lett. 42 112-5

[4] Zewail A H 2000 Femtochemistry: atomic-scale dynamics of the chemical bond J. Phys. Chem. A 104 5660-94

[5] Brida D et al 2013 Ultrafast collinear scattering and carrier multiplication in graphene Nat. Commun. 41987 
[6] Soavi G, Grupp A, Budweg A, Scotognella F, Hefner T, Hertel T, Lanzani G, Leitenstorfer A, Cerullo G and Brida D 2015 Below-gap excitation of semiconducting single-wall carbon nanotubes Nanoscale 7 18337-42

[7] Trushin $\mathrm{M}$ et al 2015 Ultrafast pseudospin dynamics in graphene Phys. Rev. B 92165429

[8] Sutter D H, Steinmeyer G, Gallmann L, Matuschek N, Morier-Genoud F, Keller U, Scheuer V, Angelow G and Tschudi T 1999 Semiconductor saturable-absorber mirrorassisted Kerr-lens mode-locked Ti:sapphire laser producing pulses in the two-cycle regime Opt. Lett. 24631

[9] Morgner U, Kärtner F X, Cho S H, Chen Y, Haus H A, Fujimoto J G, Ippen E P, Scheuer V, Angelow G and Tschudi T 1999 Sub-two-cycle pulses from a Kerr-lens mode-locked Ti:sapphire laser Opt. Lett. 24411

[10] Baltuska A, Fuji T and Kobayashi T 2002 Visible pulse compression to $4 \mathrm{fs}$ by optical parametric amplification and programmable dispersion control Opt. Lett. 27 306-8

[11] Wilhelm T, Piel J and Riedle E 1997 Sub-20 fs pulses tunable across the visible from a blue-pumped single-pass noncollinear parametric converter Opt. Lett. 221494

[12] Brida D, Manzoni C, Cirmi G, Marangoni M,

De Silvestri S and Cerullo G 2007 Generation of broadband mid-infrared pulses from an optical parametric amplifier Opt. Express 1515035

[13] Brida D, Cirmi G, Manzoni C, Bonora S, Villoresi P, De Silvestri S and Cerullo G 2008 Sub-two-cycle light pulses at $1.6 \mu \mathrm{m}$ from an optical parametric amplifier $O p t$. Lett. 33741

[14] Fu Y, Takahashi E J and Midorikawa K 2015 High-energy infrared femtosecond pulses generated by dual-chirped optical parametric amplification Opt. Lett. 405082

[15] Yin Y, Ren X, Chew A, Li J, Wang Y, Zhuang F, Wu Y and Chang Z 2017 Generation of octave-spanning mid-infrared pulses from cascaded second-order nonlinear processes in a single crystal Sci. Rep. 711097

[16] Yin Y, Li J, Ren X, Zhao K, Wu Y, Cunningham E and Chang Z 2016 High-efficiency optical parametric chirpedpulse amplifier in BiB_3O_6 for generation of $3 \mathrm{~mJ}$, twocycle, carrier-envelope-phase-stable pulses at $17 \mu \mathrm{m} \mathrm{Opt}$. Lett. 411142

[17] Kessel A, Trushin S A, Karpowicz N, Skrobol C, Klingebiel S, Wandt C and Karsch S 2016 Generation of multi-octave spanning high-energy pulses by cascaded nonlinear processes in BBO Opt. Express 245628

[18] Baum P, Lochbrunner S and Riedle E 2004 Tunable sub-10 fs ultraviolet pulses generated by achromatic frequency doubling Opt. Lett. 29 1686-8

[19] Petrov V, Rotermund F, Noack F and Schunemann P 1999 Femtosecond parametric generation in $\mathrm{ZnGeP}_{2}$ Opt. Lett. 24 414-6

[20] Seifert F, Petrov V and Woerner M 1994 Solid-state laser system for the generation of midinfrared femtosecond pulses tunable from 3.3 to 10 microm Opt. Lett. 19 2009-11

[21] Junginger F, Sell A, Schubert O, Mayer B, Brida D, Marangoni M, Cerullo G, Leitenstorfer A and Huber R 2010 Single-cycle multiterahertz transients with peak fields above $10 \mathrm{MV} / \mathrm{cm}$ Opt. Lett. 35 2645-7

[22] Yin Y, Chew A, Ren X, Li J, Wang Y, Wu Y and Chang Z 2017 Towards Terawatt Sub-cycle long-wave infrared pulses via chirped optical parametric amplification and indirect pulse shaping Sci. Rep. 845794

[23] Sanchez D, Hemmer M, Baudisch M, Cousin S L, Zawilski K, Schunemann P, Chalus O, Simon-Boisson C and Biegert J $20167 \mu \mathrm{m}$, ultrafast, sub-millijoule-level mid-infrared optical parametric chirped pulse amplifier pumped at $2 \mu \mathrm{m}$ Optica 3147

[24] Shirakawa A, Sakane I, Takasaka M and Kobayashi T 1999 Sub-5 fs visible pulse generation by pulse-front-matched noncollinear optical parametric amplification Appl. Phys. Lett. 74 2268-70

[25] Kobayashi T and Baltuska A 2002 Sub-5 fs pulse generation from a noncollinear optical parametric amplifier Meas. Sci. Technol. 13 1671-82

[26] Liebel M, Schnedermann C and Kukura P 2014 Sub-10 fs pulses tunable from 480 to $980 \mathrm{~nm}$ from a NOPA pumped by an Yb:KGW source Opt. Lett. 394112

[27] Cerullo G, Nisoli M, Stagira S and De Silvestri S 1998 Sub8 fs pulses from an ultrabroadband optical parametric amplifier in the visible Opt. Lett. 23 1283-5

[28] Steinmann A, Killi A, Palmer G, Binhammer T and Morgner U 2006 Generation of few-cycle pulses directly from a MHzNOPA Opt. Express 14 10627-30

[29] Homann C, Schriever C, Baum P and Riedle E 2008 Octave wide tunable UV-pumped NOPA: pulses down to $20 \mathrm{fs}$ at $0.5 \mathrm{MHz}$ repetition rate Opt. Express 16 5746-56

[30] Deng Y et al 2012 Carrier-envelope-phase-stable, 12 mJ, 15 cycle laser pulses at $21 \mu \mathrm{m}$ Opt. Lett. 374973

[31] Agnesi A, Greborio A, Pirzio F, Reali G, Aus der Au J and Guandalini A $201240 \mathrm{fs} \mathrm{Yb}(3+): \mathrm{CaGdAlO} 4$ laser pumped by a single-mode 350-mW laser diode Opt. Express 20 10077

[32] Fischer J, Heinrich A-C, Maier S, Jungwirth J, Brida D and Leitenstorfer A 2016615 fs pulses with $17 \mathrm{~mJ}$ energy generated by an $\mathrm{Yb}$ :thin-disk amplifier at $3 \mathrm{kHz}$ repetition rate Opt. Lett. 41246

[33] Huber R, Brodschelm A, Tauser F and Leitenstorfer A 2000 Generation and field-resolved detection of femtosecond electromagnetic pulses tunable up to $41 \mathrm{THz}$ Appl. Phys. Lett. 76 3191-3

[34] Brida D, Manzoni C, Cirmi G, Marangoni M, Bonora S, Villoresi P, De Silvestri S and Cerullo G 2010 Few-opticalcycle pulses tunable from the visible to the mid-infrared by optical parametric amplifiers J. Opt. 1213001

[35] Driscoll T J, Gale G M and Hache F 1994 Ti:sapphire secondharmonic-pumped visible range femtosecond optical parametric oscillator Opt. Commun. 110 638-44

[36] Brida D, Marangoni M, Manzoni C, De Silvestri S and Cerullo G 2008 Two-optical-cycle pulses in the mid-infrared from an optical parametric amplifier Opt. Lett. 332901

[37] Alfano R R and Shapiro S L 1970 Emission in the region 4000 to $7000 \AA$ via four-photon coupling in glass Phys. Rev. Lett. 24 584-7

[38] Alfano R R and Shapiro S L 1970 Observation of self-phase modulation and small-scale filaments in crystals and glasses Phys. Rev. Lett. 24 592-4

[39] Reed M K, Steiner-Shepard M K and Negus D K 1994 Widely tunable femtosecond optical parametric amplifier at $250 \mathrm{kHz}$ with a Ti:sapphire regenerative amplifier Opt. Lett. 19 1855

[40] Reed M K, Steiner-Shepard M K, Armas M S and Negus D K 1995 Microjoule-energy ultrafast optical parametric amplifiers J. Opt. Soc. Am. B 122229

[41] Reed M K, Armas M S, Steiner-Shepard M K and Negus D K $199530 \mathrm{fs}$ pulses tunable across the visible with a $100 \mathrm{kHz}$ Ti:sapphire regenerative amplifier Opt. Lett. 20605

[42] Bradler M, Baum P and Riedle E 2009 Femtosecond continuum generation in bulk laser host materials with sub$\mu \mathrm{J}$ pump pulses Appl. Phys. B 97 561-74

[43] Polli D, Brida D, Mukamel S, Lanzani G and Cerullo G 2010 Effective temporal resolution in pump-probe spectroscopy with strongly chirped pulses Phys. Rev. A 82 1-8

[44] Kane D J and Trebino R 1993 Characterization of arbitrary femtosecond pulses using frequency-resolved optical gating IEEE J. Quantum Electron. 29 571-9

[45] Petrov V, Noack F and Stolzenberger R 1997 Seeded femtosecond optical parametric amplification in the midinfrared spectral region above $3 \mu \mathrm{m}$ Appl. Opt. 361164 
[46] Pashkin A, Junginger F, Mayer B, Schmidt C, Schubert O, Brida D, Huber R and Leitenstorfer A 2013 Quantum physics with ultrabroadband and intense terahertz pulses IEEE J. Sel. Top. Quantum Electron. 198401608

[47] Vodopyanov K L and Kulevskii L A 1995 New dispersion relationships for GaSe in the $0.65-18 \mu \mathrm{m}$ spectral region Opt. Commun. 118 375-8

[48] Sell A, Leitenstorfer A and Huber R 2008 Phase-locked generation and field-resolved detection of widely tunable terahertz pulses with amplitudes exceeding $100 \mathrm{MV} / \mathrm{cm}$ Opt. Lett. 33 2767-9
[49] Fischer M P, Bühler J, Fitzky G, Kurihara T, Eggert S, Leitenstorfer A and Brida D 2017 Coherent field transients below $15 \mathrm{THz}$ from phase-matched difference frequency generation in 4H-SiC Opt. Lett. 422687

[50] Brabec C J, Gowrisanker S, Halls J J M, Laird D, Jia S and Williams S P 2010 Polymer-fullerene bulk-heterojunction solar cells Adv. Mater. 22 3839-56

[51] Louarn G, Trznadel M, Buisson J P, Laska J, Pron A, Lapkowski M and Lefrant S 1996 Raman spectroscopic studies of regioregular poly(3-alkylthiophenes) J. Phys. Chem. 100 12532-9 